\title{
Expression of the sperm fibrous sheath protein CABYR in human cancers and identification of $\alpha$-enolase as an interacting partner of CABYR-a
}

\author{
YEN-TZU TSENG ${ }^{1}$, JIUN-YI HSIA ${ }^{1,2}$, CHUN-YU CHEN $^{1}$, NIEN-TSUNG LIN ${ }^{3}$, \\ PELE CHOI-SING CHONG ${ }^{4}$ and CHIOU-YING YANG ${ }^{1}$ \\ ${ }^{1}$ Institute of Molecular biology, National Chung Hsing University, Taichung 402; \\ ${ }^{2}$ Division of Thoracic Surgery, Department of Surgery, Taichung Veterans General Hospital, Taichung 407; \\ ${ }^{3}$ Institute of Microbiology, Immunology and Molecular Medicine, Tzu Chi University, Hualien 970; \\ ${ }^{4}$ Vaccine Research and Development Center, National Health Research Institutes, Miaoli 350, Taiwan, R.O.C.
}

Received November 3, 2010; Accepted December 6, 2010

DOI: $10.3892 /$ or.2011.1165

\begin{abstract}
Calcium-binding tyrosine phosphorylation regulated protein (CABYR), a family of isoforms resulting from alternative splicing, has been identified as a cancer/testis antigen (CT88) in lung cancer and hypothesized to be a promising target for immunotherapy. Here, we report the expression of CABYR in various cancer tissues/cell lines. Expression profiles of individual isoforms were different among cancers. Furthermore, protein and mRNA levels did not correlate for individual isoforms. While CABYR-c/d were the most abundant splicing variants, CABYR-a was the predominant protein isoform. Finally, CABYR-a, but not CABYR-c, was found to interact with $\alpha$-enolase in vivo. Collectively, the data indicate that CABYR is a CT antigen widely expressed in diverse cancer cells. However, individual protein isoforms may be differentially regulated by post-transcriptional and post-translational mechanisms and may have a unique role in carcinogenesis. The protein expression pattern of various CABYR isoforms is important with regard to the consideration of using CABYR as a target antigen for the development of vaccines for cancer therapy.
\end{abstract}

Correspondence to: Dr Chiou-Ying Yang, Institute of Molecular Biology, National Chung Hsing University, 250 Kuo Kuang Road, Taichung 402, Taiwan, R.O.C.

E-mail: cyyang@dragon.nchu.edu.tw

Abbreviations: CABYR, calcium-binding tyrosine phosphorylation-regulated protein; CT, cancer/testis; DMEM, Dulbecco's modified Eagle's medium; FCS, fetal bovine serum; SDS-PAGE, sodium dodecyle sulfate-polyacrylamide gel electrophoresis

Key words: calcium-binding tyrosine phosphorylation regulated protein, cancer/testis antigen, liver cancer, esophageal cancer, $\alpha$-enolase

\section{Introduction}

Calcium-binding tyrosine phosphorylation regulated protein (CABYR), encoded by the CABYR gene located at chromosome $18 \mathrm{q} 11.2$ and chromosome 18 locus A1 of human and mouse, respectively, is a family of highly polymorphic proteins $(1,2)$. Six alternatively spliced transcripts involving two coding regions (CR-A and CR-B) have been shown to encode five protein isoforms (CABYR-a - CABYR-e) (Fig. 1) with extensive charge and mass polymorphisms in human spermatozoa (1). While proteins encoded by both CR-A and CR-B are produced during spermatogenesis, only $\mathrm{CR}-\mathrm{A}$-encoded acidic isoforms bind calcium. These isoforms appear to have a much higher molecular weight than the predicted $50-53 \mathrm{kDa}$ when assessed by SDS-PAGE $(1,3)$. Although CABYR was originally reported as a testis-specific protein localized exclusively in the sperm flagellum, which gains calcium-binding capacity when phosphorylated during capacitation (1), CABYR-c and CABYR-d are also detected in the normal human brain and also in the fetal brain and brain tumors in which the expression is more distinct than in the adult brain (4). This evidence, together with the detection of aberrant expression of CABYR-a/b and CABYR-c in lung cancers and the presence of specific $\operatorname{IgG}$ antibodies in some sera from lung cancer patients but not from the healthy donors, has defined CABYR as a novel cancer/testis (CT) antigen and a new potential target for the use in development of therapeutics for human cancer $(5,6)$.

Recently, CABYR was found in motile cilia of fallopian tubes and human bronchi in addition to the sperm fibrous sheath suggesting that it may have a role in calcium signaling pathways that regulate motility of cilia and flagella (7). CABYR-c and CABYR-d, which lack calcium binding capacity, have been demonstrated to interact with GSK3B in vitro (4). Both CABYR and GSK3ß genes were found to be up-regulated in smokers, suggesting a role of these proteins in carcinogenesis (8).

The present study was undertaken after we identified a recombinant phage displaying a peptide derived from CABYR 


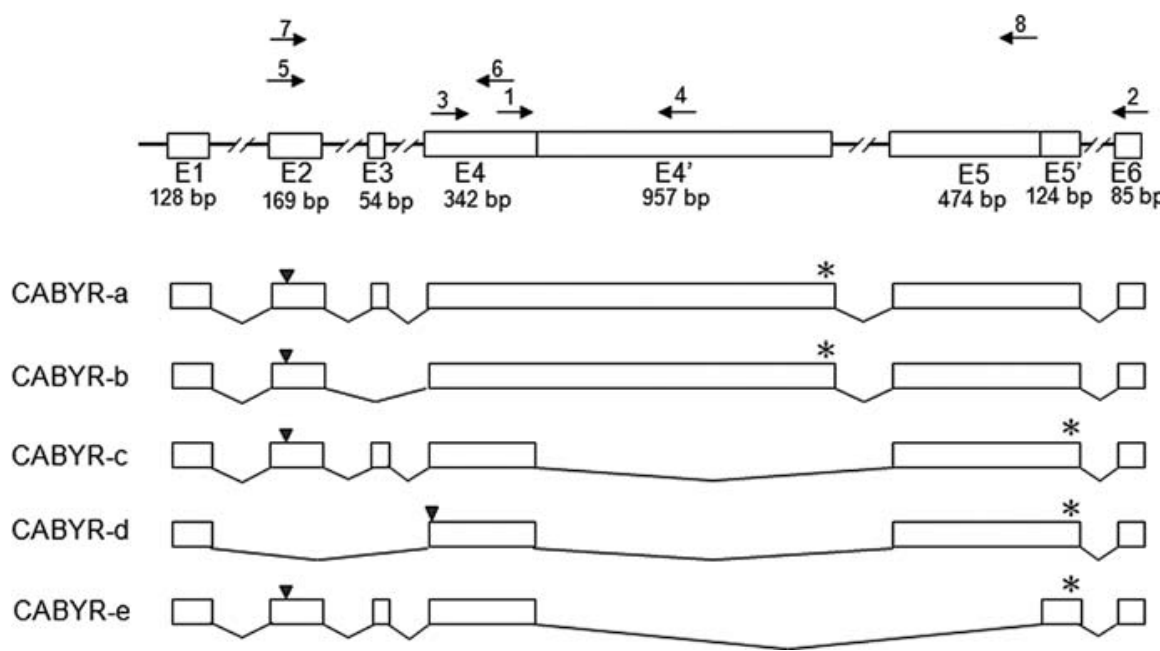

Figure 1. Diagram of CABYR genomic structure and transcript variants. Translation start codons $(\mathbf{v})$ and stop codons $(*)$ are indicated. Relative positions of the primer pairs used to detect splicing variants $(1 / 2,3 / 4$, and 5/6) and prepare the biotinylated probe (7/8) are indicated by arrows.

that was affinity-selected by a rabbit serum against hepatocellular carcinoma HepG2 cells. Here, we show that CABYR is aberrantly expressed in a wide range of human cancers. Interestingly, although the CABYR-c/d isoforms are more readily detected at the mRNA level, CABYR-a is the prevalent isoform at the protein level. In addition, we found that CABYR-a, but not CABYR-c, interacts with $\alpha$-enolase, suggesting that CABYR may have a role in modulating $\alpha$-enolase function.

\section{Materials and methods}

Cell culture and tissue specimens. DMEM supplemented with $10 \% \mathrm{FBS}$ and $50-\mu \mathrm{g} / \mathrm{ml}$ gentamycin was used to cultivate the hepatocellular carcinoma cell lines, HepG2 and Huh7, the nasopharyngeal carcinoma cell lines, NPC039, NPC076 and BM1, the human embryonic kidney cell line, 293T, the breast cancer cell line Hs578T, the lung adenocarcinoma cell lines CL1-0 and CL1-5, and the human glioblastoma-astrocytoma cell line U-87 MG. Tissue specimens were obtained from Taichung Veterans General Hospital, Taiwan and Buddhist Tzu Chi General Hospital, Hualien, Taiwan.

$R T-P C R$. Total RNA was prepared using TRIzol reagent (Invitrogen, Carlsbad, CA, USA) according to the manufacturer's instructions. RNA was reverse transcribed in a reaction mixture of $20 \mu \mathrm{l}$ containing $5 \mu \mathrm{g}$ of total RNA, $2.5 \mu \mathrm{g}$ of oligo dT15, 1 nmole of dNTP and Superscript II reverse transcriptase (Invitrogen). Normal tissue cDNA were purchased from Clontech (Mountain View, CA, USA). cDNA was amplified by PCR using Taq DNA Polymerase (Invitrogen) with $1 \mu \mathrm{l}$ of the cDNA as the template. Thermal cycle conditions were as follows: $94^{\circ} \mathrm{C}$ for $5 \mathrm{~min}$ followed by 30 cycles of $94^{\circ} \mathrm{C}$ for $1 \mathrm{~min}, 60^{\circ} \mathrm{C}$ for $2 \mathrm{~min}$ and $72^{\circ} \mathrm{C}$ for $1 \mathrm{~min}$, and then extension at $72^{\circ} \mathrm{C}$ for $10 \mathrm{~min}$. Integrity of the cDNA was verified by amplification of the $B$-actin gene. PCR products were confirmed by nucleotide sequencing or Southern hybridization. Primers used in this study are listed in Table I.
Southern hybridization. Southern hybridization was carried out essentially as previously described (9) using biotinylated CABYR DNA obtained by PCR with primers CABYR-7 and CABYR-8 (Table I, Fig. 1). PCR amplification conditions were as described above, except that the nucleotide mixtures contained $1.25 \mathrm{mM}$ biotin-11-dATP (Perkin-Elmer, Waltham, MA, USA) in addition to $2.5 \mathrm{mM}$ each of dTTP, dGTP, and $\mathrm{dCTP}$, and $1.25 \mathrm{mM}$ dATP. After hybridization, the membrane was incubated with alkaline phosphatase-conjugated streptavidin (Invitrogen) and developed using CDP-star chemiluminescence reagent (Perkin-Elmer). The hybridization signals were then detected by an ImageMaster VDS-CL system (GE Healthcare, Piscataway, NJ, USA).

Plasmids. For nucleotide sequencing, PCR products were cloned into pGEM-T Easy vector (Promega Co., Madison, WI, USA). To produce recombinant CABYR proteins for immunization, the complete coding regions of CABYR-a (GenBank ID: HM594166) and CABYR-c (GenBank ID: HM594168) were PCR amplified from NPC076 cell cDNA with primer pairs CABYR-F/CABYR-a-R and CABYR-F/ CABYR-c-R, respectively, and cloned into pET21b (Merck KGaA, Darmstadt, Germany) to generate pET-CABYR-a and pET-CABYR-c. To express Flag-tagged CABYR in 293T cells, DNA fragments encoding the full length CABYR-a were amplified from pET-CABYR-a using primer pairs CRA-F/ CRA-R, while CABYR-c was amplified from pET-CABYR-c with primer pairs CRA-F/CRB-R. Both amplification products were cloned into the EcoRI/NotI site of pcDNA-Flagß to generate Flag-CABYR-a and Flag-CABYR-c.

Antibodies. Anti-CABYR antibodies were prepared by immunization of rabbits with E. coli produced His-tagged CABYR. Briefly, pET-CABYR-a and pET-CABYR-c plasmids were transformed separately into E. coli $\mathrm{BL} 21(\mathrm{DE} 3)$ and the transformants were grown in LB for $3 \mathrm{~h}$, after which expression was induced with IPTG $(1 \mathrm{mM})$. His-tagged CABYR-a and CABYR-c proteins were purified using a HisBind purification kit (Merck KGaA) following the manufacturer's protocol. 
Table I. Primer sequence used in this study.

\begin{tabular}{|c|c|}
\hline Primer & Sequence $^{a}$ \\
\hline \multicolumn{2}{|l|}{ RT-PCR ${ }^{b}$} \\
\hline CABYR-1 & 5'-GCTCAGCTTGCTGCTCAGATG-3' \\
\hline CABYR-2 & 5'-TCCCTGAAATGTTGACCCAG-3' \\
\hline CABYR-3 & 5'-cgaaTtCCCAGATGGAAAAATCTAC-3' \\
\hline CABYR-4 & 5'-gcggccgcTcaTTCTGGCAGTTGCTC-3' \\
\hline CABYR-5 & 5'-agaatTgCCAAAATGATTTCTTCAAAGCCC-3' \\
\hline CABYR-6 & 5'-TAACATCTGAGCAGCAAGCTG-3' \\
\hline$ß$-actin-F & 5'-GCTTGACTCAGGATTTAAAAACTGGAACGG-3' \\
\hline B-actin-R & 5'-TATTCAACTGGTCTCAAGTCAGTGTACAGG-3' \\
\hline \multicolumn{2}{|c|}{ Probe synthesis ${ }^{b}$} \\
\hline CABYR-7 & 5'-tggatccAATGATTTCTTCAAAGCCC-3' \\
\hline CABYR-8 & 5'-tAagcTTGTCACCAGATCCTGAAC-3' \\
\hline \multicolumn{2}{|c|}{ His-tagged expression plasmid construction } \\
\hline CABYR-F & 5'-cgaATtCTGCCAAAATGATTTCTT-3' \\
\hline CABYR-a-R & 5'-tAagcTtTTCAGCTGTTGATTCCCC-3' \\
\hline CABYR-c-R & 5'-aacaagcttGTTTTCAGTTTCTGC-3' \\
\hline \multicolumn{2}{|c|}{ Flag-tagged expression plasmid construction } \\
\hline CRA-F & 5'-agaatTcCCAAAATGATTTCTTCAAAGCCC-3' \\
\hline CRA-R & 5'-tgcggccgCTTATTCAGCTGTTGATTCCCC-3' \\
\hline CRB-R & 5'-tgcggccGcTCAGTTTTCAGTTTCTGCTTTGCG-3' \\
\hline
\end{tabular}

aThe mismatched nucleotides are in lower case and restriction enzyme recognition sites are underlined. ${ }^{\mathrm{b}}$ The relative positions of CABYR specific primers used in RT-PCR and probe synthesis are shown in Fig. 1.

After extensive dialysis against PBS, the purity was monitored by SDS-PAGE and the protein concentration was determined using Protein Assay Dye Reagent Concentrate (Bio-Rad, Hercules, CA, USA). Rabbits were immunized subcutaneously with recombinant CABYR-a or CABYR-c proteins (150 $\mu \mathrm{g}$; emulsified with complete Freund's adjuvant for the first injection and incomplete Freund's adjuvant for the boost) four times at 2-week intervals. Rabbit anti-human $\alpha$-enolase (H300) and mouse monoclonal anti-ß-actin (Ac15) antibodies were obtained from Santa Cruz Biotechnology (Santa Cruz, CA, USA) and Novus Biologicals (Littleton, CO, USA), respectively.

Western blotting. Cells were lysed with NP-40 lysis buffer (1\% NP-40, $150 \mathrm{mM} \mathrm{NaCl}, 50 \mathrm{mM}$ Tris- $\mathrm{HCl}, \mathrm{pH}$ 8.0) and tissues were pulverized using liquid nitrogen. Proteins were extracted with modified Camiolo buffer $(0.25 \%$ Triton X-100, $300 \mathrm{mM}$ $\mathrm{NaCl}, 10 \mathrm{mM}$ EDTA, $75 \mathrm{mM}$ potassium acetate, $100 \mathrm{mM}$ L-arginine, $\mathrm{pH}$ 7.4) (10). Both buffers contained protease inhibitor cocktail including $1.04 \mathrm{mM}$ AEBSF, $0.85 \mu \mathrm{M}$ aprotinin, $40 \mu \mathrm{M}$ bestatin, $14 \mu \mathrm{M}$ E-64, $20 \mu \mathrm{M}$ leupeptin, $15 \mu \mathrm{M}$ pepstatin (Sigma-Aldrich, St. Louis, MO, USA). Normal liver and testis proteins were purchased from Clontech. Western blotting was performed following standard procedures (9).
The blots were probed with appropriate primary antibodies and detected with alkaline phosphatase-conjugated goat antirabbit or anti-mouse IgG secondary antibodies. The immunoreactive band was visualized by developing with BCIP and NBT (5-bromo-4-chloro-3-indolyl phosphate/nitroblue tetrazolium).

Identification of CABYR-interacting protein(s) by immunoprecipitation. Flag-tagged expression plasmids were transiently transfected into $293 \mathrm{~T}$ cells using jetPEI reagent (Polyplus-transfection Inc., NY, USA) according to the manufacturer's instructions. Briefly, 293T cells were cultured in $10-\mathrm{cm}$ diameter cell culture dishes until they reached $60 \%$ confluency and then $20 \mu \mathrm{g}$ of the Flag-tagged expression plasmid was added to each dish. After $48 \mathrm{~h}$, cells were harvested and lysed with NP-40 lysis buffer. After $10 \mathrm{~min}$ on ice, lysed cells were centrifuged $(16,000 \mathrm{x} \mathrm{g})$ for $15 \mathrm{~min}$ at $4^{\circ} \mathrm{C}$ to separate the cytoplasmic and nuclear fractions. The supernatants were incubated with anti-Flag M2 agarose (Sigma-Aldrich) for $2 \mathrm{~h}$ with shaking at $4^{\circ} \mathrm{C}$. The proteinbound M2-agarose beads were washed three times with NP-40 lysis buffer, and the immunoprecipitates were eluted 3 times with $0.1 \mathrm{M}$ glycine $/ \mathrm{HCl}(\mathrm{pH} 3.5)$ and neutralized immediately with $1 \mathrm{M}$ Tris- $\mathrm{HCl}(\mathrm{pH} 9.5)$. Eluted samples 

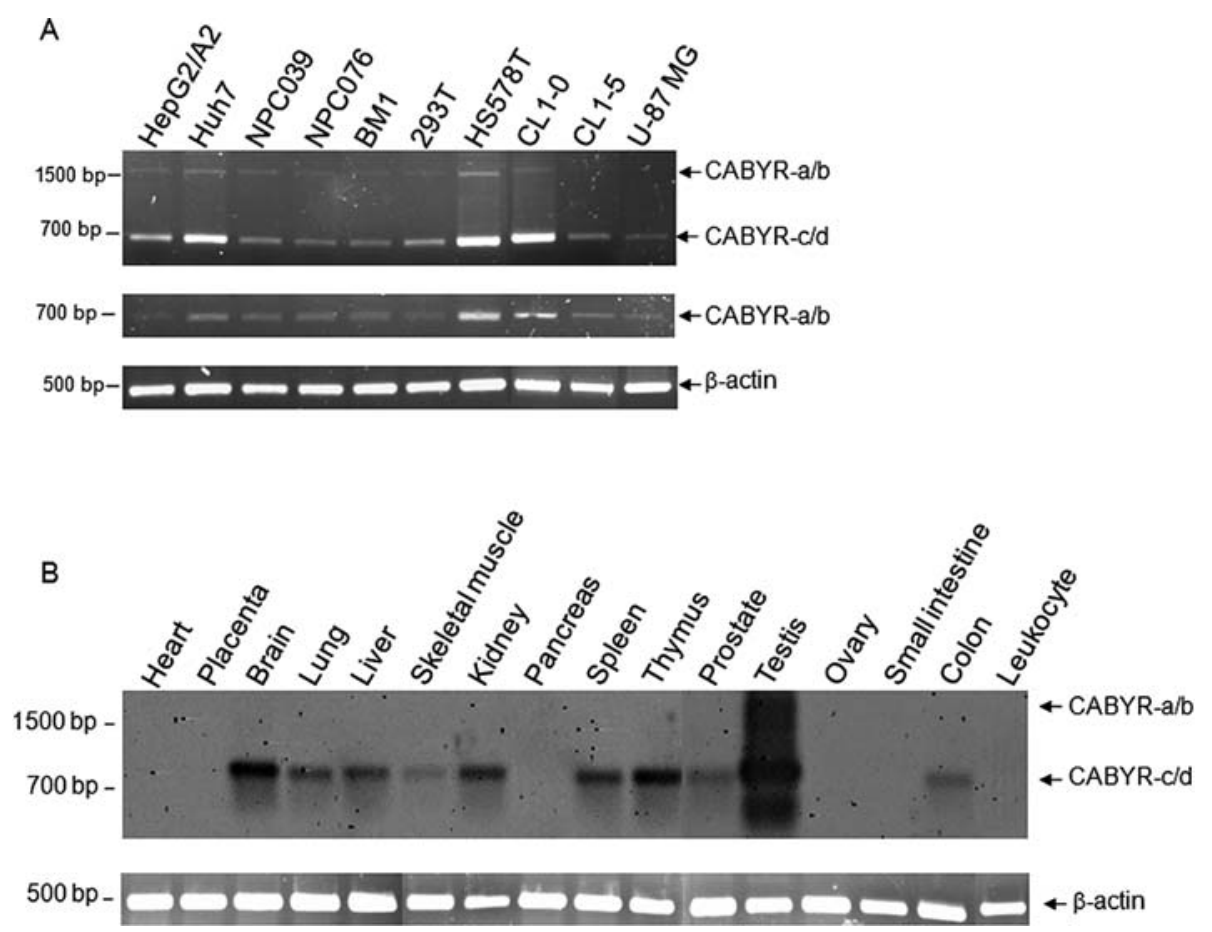

Figure 2. RT-PCR analysis for expression of CABYR transcript variants in cancer cell lines using isoform-conserved primers (top) and CABYR-a/b specific primers (middle) detected by ethidium bromide staining (A) and normal tissues using isoform-conserved primers detected by Southern blotting with CABYRspecific probe (B). $\beta$-actin was used as an internal control.

Table II. CABYR mRNA expression in liver, lung and esophageal cancers.

Expression frequency (no. positive/no. tested)

\begin{tabular}{|c|c|c|c|c|c|}
\hline \multicolumn{2}{|c|}{ CABYR-a/b } & \multicolumn{2}{|c|}{ CABYR-c/d } & \multicolumn{2}{|c|}{ CABYR-e ${ }^{a}$} \\
\hline $\begin{array}{l}\text { Tumor tissue } \\
(\%)\end{array}$ & $\begin{array}{c}\text { Adjacent tissue } \\
(\%)\end{array}$ & $\begin{array}{c}\text { Tumor tissue } \\
(\%)\end{array}$ & $\begin{array}{c}\text { Adjacent tissue } \\
(\%)\end{array}$ & $\begin{array}{l}\text { Tumor tissue } \\
(\%)\end{array}$ & $\begin{array}{l}\text { Adjacent tissue } \\
(\%)\end{array}$ \\
\hline $12(3 / 25)$ & $0(0 / 13)$ & $80(20 / 25)$ & $38.5(5 / 13)$ & $16(4 / 25)$ & $0(0 / 13)$ \\
\hline $43.8(7 / 16)$ & $6.3(1 / 16)$ & $87.5(14 / 16)$ & $93.8(15 / 16)$ & $0(0 / 16)$ & $0(0 / 16)$ \\
\hline $16.6(7 / 42)$ & $6.3(2 / 32)$ & $59.5(25 / 42)$ & $31.3(10 / 32)$ & $4.8(2 / 42)$ & $6.3(2 / 32)$ \\
\hline
\end{tabular}

${ }^{a} A 1 l$ CABYR-e positive specimens were CABYR-a/b positive except for one lung tumor tissue.

were separated by SDS-PAGE, and proteins were detected by Western blotting or by silver staining as previously described (11).

\section{Results}

Expression of CABYR mRNA in human cell lines and in tissues from cancer patients. With the finding that a phage displaying a peptide derived from CABYR was affinity-selected by anti-HepG2 antiserum, we examined the expression of CABYR in the HepG2 cells together with a panel of other human cell lines by RT-PCR using the isoform-conserved primers, CABYR-1 and CABYR-2 (Fig. 1, Table I). Distinct $0.65 \mathrm{~kb}$ bands and faint $1.6 \mathrm{~kb}$ bands corresponding to the sizes of CABYR-c/d and CABYR-a/b transcripts, respectively, were obtained (Fig. 2A). The identity of these two DNA fragments as CABYR was verified by nucleotide sequencing of the products (GenBank ID: HM594167, HM594163) and by Southern hybridization of all samples (data not shown). CABYR-a/b mRNA could be detected in almost all tested cells with relative intensities significantly lower than the CABYR-c/d. To minimize the effect of target length on amplification efficiency, we used CABYR-a/b specific PCR primers that generated $0.65-\mathrm{kb}$ products (CABYR-3 and CABYR-4) (Fig. 2A). The levels of CABYR-c/d were consistently higher than the CABYR-a/b based on relative band intensities compared to the internal control, B-actin.

Next, we examined the expression of CABYR mRNA in tumors and adjacent tissues from liver, esophageal, and lung cancer patients using the isoform-conserved primers, with commercially available normal tissue cDNA as a control. In addition to CABYR-a/b and CABYR-c/d (verified by 
A

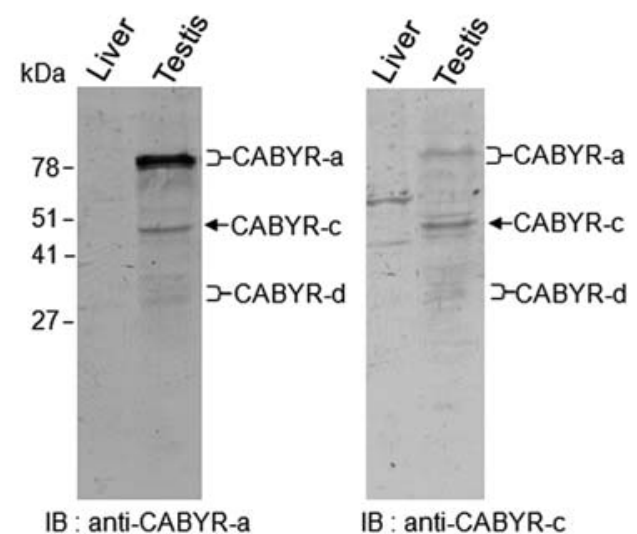

B

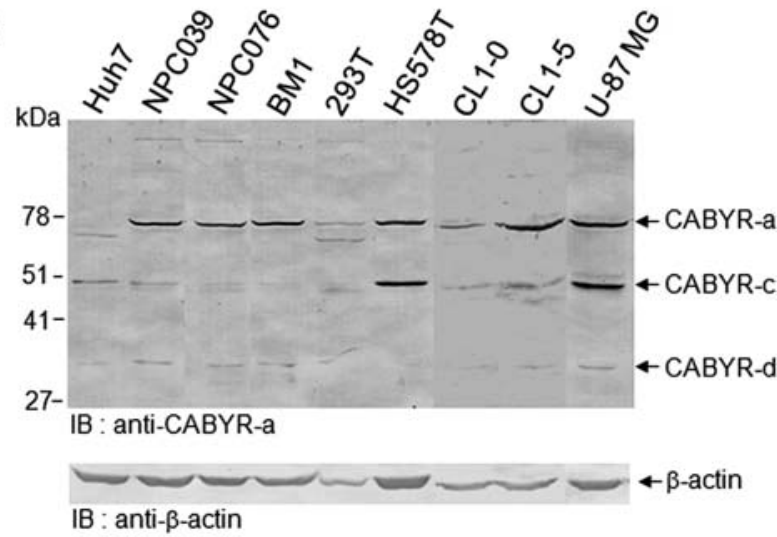

C

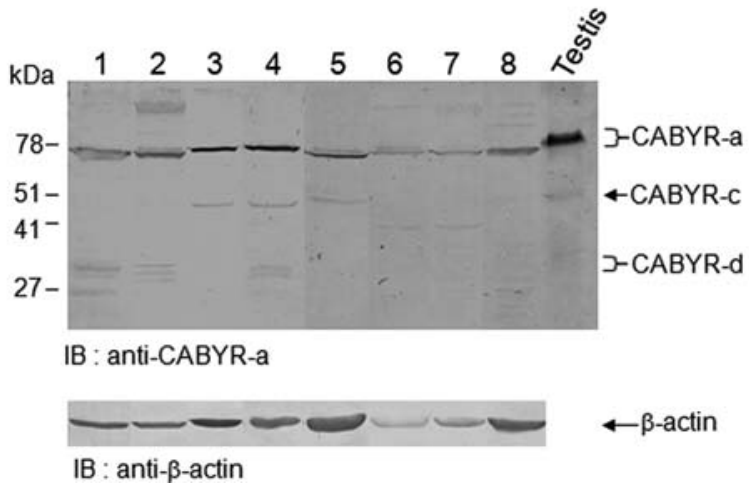

Figure 3. Western blot analysis of CABYR protein isoform expression in normal human tissues (A), cancer cell lines (B), and tumor tissues from liver cancer patients (C). Primary antibodies used are as indicated.

Southern hybridization), PCR products corresponding to the CABYR-e transcript (verified by nucleotide sequencing) (GenBank ID: HM594169) were obtained in some tissue samples (data not shown). CABYR-a/b mRNA was detected more frequently in tumor tissues than in adjacent noncancerous tissues but CABYR-c/d and CABYR-e had different patterns of expression (Table II). CABYR-c/d was more frequently detected in tumor tissues of lung and esophageal cancers but was also detectable in most liver tissues from liver cancer patients. In contrast, CABYR-e was detected in lung and esophageal cancers but not in liver cancer. Interestingly, CABYR-a/b or CABYR-e were only detected in CABYR-c/d positive samples. It is noteworthy that CABYR-c/d but not CABYR-a/b was detected in several normal tissues other than testis and brain, including lung and liver in one of the two batches of tissue from Clontech (Fig. 2B).

Expression of CABYR protein in liver cancer tissues and cancer cell lines. To investigate whether the RNA expression translates to a biologically significant level of protein, we prepared antisera against the recombinant CABYR-a and CABYR-c proteins. The recombinant proteins were recognized by anti-HepG2 serum, confirming the presence of CABYRspecific antibodies in this serum (data not shown). After verifying their specificity, the sera against the recombinant CABYR were used to examine normal testis and liver proteins by Western blot analysis. Three groups of immunoreactive bands, corresponding to CABYR-a/b, CABYR-c and CABYR-d, were detected only in normal testis but were not detected in normal liver protein by either antiserum (Fig. 3A). Interestingly, although the mRNA level of CABYR-c/d was significant higher than that of CABYR-a/b in normal testis (Fig. 2B), it appears that CABYR-a/b were at least as prevalent as CABYR-c/d proteins.

Because of the lower background, we used anti-CABYR-a to examine the CABYR protein expression in liver tissues from cancer patients as well as in cancer cell lines including U-87 MG, which had previously been shown to express CABYR-c and CABYR-d isoforms (4). No signal was detected in immunoblots probed with pre-immune serum (data not shown). Immunoreactive bands of sizes corresponding to CABYR-a/b, CABYR-c and CABYR-d were detected in human cell lines (Fig. 3B) as well as the liver tumor tissues (Fig. 3C). Surprisingly, we found that the CABYR-a/b proteins of cancer origin migrated slightly faster than the corresponding testis protein and was detected in tissue samples without detectable CABYR-a/b transcripts. The expression profiles of CABYR-c and CABYR-d proteins were more heterogeneous and also did not correlate with the mRNA levels. The data suggest that CABYR-a/b protein may have a longer half-life than the CABYR-c/d and that their expression is differentially regulated.

CABYR-a and CABYR-b are similar in size and $p I$. To clarify the identity of the immunoreactive bands around the 78-kDa marker, we performed PCR using primers CABYR-5 and CABYR-6 (Fig. 1, Table I) to amplify the cDNA from cancer cell lines. A fragment corresponding to CABYR-a/c but not to CABYR-b transcripts was obtained in all tested cells (data not shown), suggesting that the immunoreactive bands migrated around $78 \mathrm{kDa}$ are the products of the CABYR-a transcript. Finally, we determined the nucleotide sequences of the RT-PCR products amplified from the NPC076 cells using the primers CABYR-F and CABYR-a-R (Table I). The deduced amino acid sequences were identical to the testis CABYR-a, suggesting that the size variation may due to post-translational modifications.

CABYR-a interacts with $\alpha$-enolase. In an initial attempt to elucidate the biological function of CABYR, we used coimmunoprecipitation to isolate potential CABYR binding partner(s). Because the anti-CABYR sera were not able to recognize the CABYR proteins in their native form, we trans- 


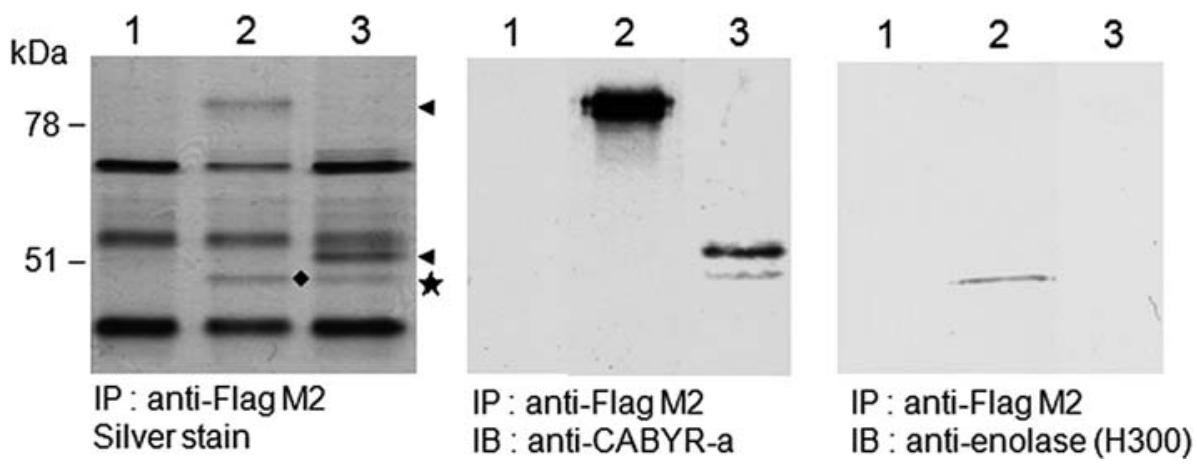

Figure 4. Demonstration of the interaction between CABYR-a and $\alpha$-enolase by immunoprecipitation and Western blot analysis. Silver staining (left panel) and Western blots of the anti-Flag immunoprecipitates from 293T cells transfected with pcDNA-Flagß (1), Flag-CABYR-a (2) and Flag-CABYR-c (3). $\triangleleft$, Flag-tagged CABYR; $\star$, endogenous CABYR;,$\alpha$-enolase. Primary antibodies used are as indicated.

fected Flag-tagged expression plasmids into $293 \mathrm{~T}$ cells and immunoprecipitated the cytoplasmic fraction with an antiFlag antibody. After SDS-PAGE analysis, a band located near $50 \mathrm{kDa}$, in addition to the CABYR proteins, was detected in the immunoprecipitates of the Flag-CABYR-a expressing cells (Fig. 4). The $50-\mathrm{kDa}$ protein was recovered and subjected to tandem mass spectrometric analysis. It was identified as $\alpha$-enolase. This was further confirmed by Western blot analysis of the immunoprecipitates with an anti$\alpha$-enolase antibody (Fig. 4). Notably, such a protein-protein interaction was not observed in in vitro pull down assays (data not shown), suggesting that the interaction is conformational dependent or that it occurs only under certain conditions.

\section{Discussion}

Due to their restricted expression profile in normal tissues, CT antigens are considered to be ideal candidates for therapeutic cancer vaccines $(12,13)$. Currently, there are over 130 CT gene families in the CT database (http://www.cta. lncc.br) based on their mRNA expression profile. Recently, sperm fibrous sheath proteins, CABYR and Sp17, were proposed to be a new class of target antigens useful for developing cancer vaccines. One advantage of these two $\mathrm{CT}$ antigens lies in their post-meiotic expression pattern, which may reduce the risk of permanent injury to the testis of patients (5). Sp17 has been reported in various malignancies (14-16). In contrast, the expression of CABYR has only been examined in brain and lung cancers $(4,6)$. In this study, we analyzed the expression of CABYR in tissues from liver, esophagus, and lung and in human cell lines of different histological origin at the mRNA and protein levels by RTPCR and Western blot analysis, respectively. We found that CABYR-a/b and CABYR-c/d were detected in all cancer cell lines and were differentially expressed in tissues from cancer patients suggesting that CABYR may be a CT gene widely expressed in different cancer types.

However, expression profiles of each isoform may be different among cancers. For example, CABYR-e was not detected in any liver specimens but in $16 \%$ of lung tumor specimens and in 4.8 and $6.3 \%$ of esophageal tumor and adjacent tissues, respectively. In addition, CABYR-c/d was detected in a high percentage of liver tumor and adjacent tissues and more frequently in tumors than in adjacent tissues of lung and esophageal cancer (Table II). Our current data suggest that CABYR is widely expressed in different cancers and that the expression profile may reflect changes in cell status. This assumption is supported by previous finding that CABYR exhibits reversible, increased expression with active smoking (8). Furthermore, the occasional detection of CABYR-c/d mRNA in normal tissues by us (Fig. 2B) and others $(4,6,7)$ may also be due to the expression of CABYR in normal tissues may be controlled by other factors, resulting in a mosaic pattern of expression. It is noteworthy that we found that 7 out of 8 CABYR-e positive specimens were both CABYR-a/b positive and CABYR-c/d positive. The presence of a specific CABYR mRNA may correlate with cellular abnormality or with histological type, as has been reported in lung cancer (6). In either case, CABYR may have potential as a diagnostic marker and therefore deserves to be studied further.

In addition to the mRNA expression, we also examined the expression of CABYR protein by Western blotting using rabbit anti-CABYR-a and found that there was no correlation between mRNA and the corresponding protein expression (Figs. 2 and 3). For example, the CABYR-a protein isoform was detected in all tested samples including mRNA-negative liver specimens but the opposite was found for CABYR-c/d. This discrepancy may be the result of variation in the liver tissue sampling area or of the differential sensitivity of the antiserum used, which exhibits higher avidity to CABYR-a than CABYR-c/d (Fig. 3A). However, the observation that Hs578T and U-87 MG cells contained high levels of CABYR-c protein ruled out the latter possibility. Together, the data indicate that the different isoforms of CABYR gene product may be regulated differentially. It has been reported that in human mRNAs length is positively correlated with the mRNA decay rate (17) and longer proteins are relatively more stable (18). Thus, our results implicate that CABYR-a/b is more stable than CABYR-c/d at protein level. Notably, a pseudogene with 50 and $85 \%$ homology at the RNA level to CABYR-a and CABYR-c, respectively, is present on chromosome 3 (GenBank ID: NG_002394). It will be of interest to know whether this pseudogene plays a function in the differential regulation of the CABYR protein expression. 
It has been demonstrated in vitro that $\mathrm{CABYR}$ interacts weakly with radial spoke protein 3 (RSP3) via its RII dimerization domain (7) and that CABYR-c/d are substrates of GSK3B (4). In this study, we showed that CABYR-a interacts with $\alpha$-enolase in vivo but not in vitro. It is possible that this interaction depends on appropriate modifications or/and physiological conditions such as phosphorylation or/ and $\mathrm{Ca}^{2+}$ concentration (19-21). It has been proposed that in the cytoplasm of tumor cells, $\alpha$-enolase and other glycolytic enzymes interact with the cytoskeleton system and that such association may provide ATP to promote the migration of tumor cells (22-24). Furthermore, $\alpha$-enolase can also be expressed on the cell surface depending on the activation status or pathophysiological conditions (25). Up-regulation of $\alpha$-enolase is strongly associated with disease malignancy (26-28). Therefore, it is possible that CABYR-a plays a role in the regulation of cellular energy or acts on the signal transducing pathway that is initiated upon binding of plasminogen to $\alpha$-enolase. Our data, together with the finding that CABYR-c/d isoforms but not CABYR-a interact with GSK3ß (4), indicate that different isoforms may have a differential roles during carcinogenesis.

In conclusion, we have detected aberrant expression of the CABYR gene in tissues from lung, liver, and esophageal cancer patients, with CABYR-c/d transcripts being found most frequently and abundantly, followed by CABYR-a/b and then CABYR-e. However, expressions of individual isoforms do not correlate with mRNA levels, suggesting that individual protein isoforms may be differentially regulated by post-transcriptional and post-translational mechanisms. The detection of CABYR-c/d transcripts in several normal tissues (Fig. 2B), together with the previous finding that CABYR protein is detected in the motile cilia of human bronchi and fallopian tubes (7), indicate that it is important to understand the expression profile of individual protein isoforms in normal tissues. Caution should therefore be taken in using CABYR protein as an immunotherapeutic target until isoform expression is better understood. In addition, the identification of a protein that interacts with CABYR in vivo provides a clue to deciphering the functions of this protein.

\section{Acknowledgements}

This study was supported in part by Liver Disease Prevention and Treatment Research Foundation, Taiwan. Yen-Tzu Tseng was awarded a PhD Student Fellowship from 2005 to 2008 by National Health Research Institute, Miaoli, Taiwan. We thank Drs Yu-Sun Chang and Jeremy J.W. Chen for providing the NPC cell lines and lung cancer cell lines, respectively, and Dr Wen-Ming Yang for pcDNA-Flagß vector.

\section{References}

1. Naaby-Hansen S, Mandal A, Wolkowicz MJ, et al: CABYR, a novel calcium-binding tyrosine phosphorylation-regulated fibrous sheath protein involved in capacitation. Dev Biol 242: 236-254, 2002.

2. Sen B, Mandal A, Wolkowicz MJ, et al: Splicing in murine CABYR and its genomic structure. Gene 310: 67-78, 2003.

3. Kim YH, Jha KN, Mandal A, et al: Translation and assembly of CABYR coding region $\mathrm{B}$ in fibrous sheath and restriction of calcium binding to coding region A. Dev Biol 286: 46-56, 2005 .
4. Hsu HC, Lee YL, Cheng TS, Howng SL, Chang LK, Lu PJ and Hong YR: Characterization of two non-testis-specific CABYR variants that bind to GSK3beta with a proline-rich extensin-like domain. Biochem Biophys Res Commun 329: 1108-1117, 2005.

5. Chiriva-Internati M, Cobos E, Da Silva DM and Kast WM: Sperm fibrous sheath proteins: a potential new class of target antigens for use in human therapeutic cancer vaccines. Cancer Immun 8: 8-12, 2008.

6. Luo C, Xiao X, Liu D, et al: CABYR is a novel cancer-testis antigen in lung cancer. Clin Cancer Res 13: 1288-1297, 2007.

7. Newell AE, Fiedler SE, Ruan JM, et al: Protein kinase A RIIlike (R2D2) proteins exhibit differential localization and AKAP interaction. Cell Motil Cytoskeleton 65: 539-552, 2008.

8. Chari R, Lonergan KM, Ng RT, MacAulay C, Lam WL and Lam S: Effect of active smoking on the human bronchial epithelium transcriptome. BMC Genomics 8: 297, 2007.

9. Sambrook J, Fritch EF and Maniatis T: Molecular Cloning: A Labratory Manual. 3rd edition. Cold Spring Harbor Laboratory Press, Cold Spring Harbor, NY, 2001.

10. Camiolo SM, Siuta MR and Madeja JM: Improved medium for extraction of plasminogen activator from tissue. Prep Biochem 12: 297-305, 1982.

11. Hsu CA, Lin WR, Li JC, et al: Immunoproteomic identification of the hypothetical protein NMB1468 as a novel lipoprotein ubiquitous in Neisseria meningitidis with vaccine potential. Proteomics 8: 2115-2125, 2008.

12. Caballero OL and Chen YT: Cancer/testis (CT) antigens: potential targets for immunotherapy. Cancer Sci 100: 2014-2021, 2009.

13. Scanlan MJ, Gure AO, Jungbluth AA, Old LJ and Chen YT: Cancer/testis antigens: an expanding family of targets for cancer immunotherapy. Immunol Rev 188: 22-32, 2002.

14. Grizzi F, Gaetani P, Franceschini B, et al: Sperm protein 17 is expressed in human nervous system tumours. BMC Cancer 6: 23, 2006.

15. Lim SH, Wang Z, Chiriva-Internati M and Xue Y: Sperm protein 17 is a novel cancer-testis antigen in multiple myeloma. Blood 97: 1508-1510, 2001.

16. Straughn JM Jr, Shaw DR, Guerrero A, et al: Expression of sperm protein 17 (Sp17) in ovarian cancer. Int J Cancer 108: 805-811, 2004.

17. Feng L and Niu DK: Relationship between mRNA stability and length: an old question with a new twist. Biochem Genet 45: 131-137, 2007.

18. Yen HC, Xu Q, Chou DM, Zhao Z and Elledge SJ: Global protein stability profiling in mammalian cells. Science 322: 918-923, 2008.

19. Manni S, Mauban JH, Ward CW and Bond M: Phosphorylation of the cAMP-dependent protein kinase (PKA) regulatory subunit modulates PKA-AKAP interaction, substrate phosphorylation, and calcium signaling in cardiac cells. J Biol Chem 283: 24145-24154, 2008.

20. Sadik G, Tanaka T, Kato K, Yamamori H, Nessa BN, Morihara T and Takeda M: Phosphorylation of tau at Ser214 mediates its interaction with 14-3-3 protein: implications for the mechanism of tau aggregation. J Neurochem 108: 33-43, 2009.

21. Sakiyama H, Wynn RM, Lee WR, et al: Regulation of nuclear import/export of carbohydrate response element-binding protein (ChREBP): interaction of an alpha-helix of ChREBP with the 14-3-3 proteins and regulation by phosphorylation. J Biol Chem 283: 24899-24908, 2008

22. Keller A, Peltzer J, Carpentier G, et al: Interactions of enolase isoforms with tubulin and microtubules during myogenesis. Biochim Biophys Acta 1770: 919-926, 2007.

23. Kim JW and Dang CV: Multifaceted roles of glycolytic enzymes. Trends Biochem Sci 30: 142-150, 2005.

24. Volker KW, Reinitz CA and Knull HR: Glycolytic enzymes and assembly of microtubule networks. Comp Biochem Physiol B Biochem Mol Biol 112: 503-514, 1995.

25. Pancholi V: Multifunctional alpha-enolase: its role in diseases. Cell Mol Life Sci 58: 902-920, 2001

26. Ejeskar K, Krona C, Caren H, Zaibak F, Li L, Martinsson T and Ioannou PA: Introduction of in vitro transcribed ENO1 mRNA into neuroblastoma cells induces cell death. BMC Cancer 5: $161,2005$.

27. He P, Naka T, Serada S, et al: Proteomics-based identification of alpha-enolase as a tumor antigen in non-small lung cancer. Cancer Sci 98: 1234-1240, 2007.

28. Maris JM and Matthay KK: Molecular biology of neuroblastoma. J Clin Oncol 17: 2264-2279, 1999. 